\title{
ON QUASIAFFINE TRANSFORMS OF UNILATERAL SHIFTS
}

\author{
KATSUTOSHI TAKAHASHI
}

\begin{abstract}
It is shown that if a contraction $T$ is a quasiaffine transform of a unilateral shift of finite multiplicity, then both the approximate point spectrum and the essential spectrum of $T$ coincide with the unit circle.
\end{abstract}

Let $T_{1}$ and $T_{2}$ be bounded linear operators on Hilbert spaces $\mathscr{H}_{1}$ and $\mathscr{H}_{2}$, respectively. The operator $T_{1}$ is said to be a quasiaffine transform of $T_{2}$ if there exists an injection $X: H_{1} \rightarrow H_{2}$ with dense range such that $X T_{1}=T_{2} X$, and this relation of $T_{1}$ and $T_{2}$ is denoted by $T_{1} \prec T_{2}$. If both $T_{1} \prec T_{2}$ and $T_{2} \prec T_{1}$, then we say that $T_{1}$ and $T_{2}$ are quasisimilar. We also write $T_{1} \prec^{i} T_{2}$ to denote that there exists an injection $X$ such that $X T_{1}=T_{2} X$. In this note we treat contractions on a separable Hilbert space which are quasiaffine transforms of unilateral shifts. Examples of such contractions are given by the results of Clary (see [3, pp. 448-450]) and [7, 8, 10, $12,13,14]$. On the other hand, the results of $[1,11]$ show that such contractions inherit the reflexivity and the bicommutant property of unilateral shifts.

Suppose that a contraction $T$ is a quasiaffine transform of a unilateral shift $S$. Then it is easily seen that every $\lambda$ in the open unit disc $\mathbf{D}=\{\lambda:|\lambda|<1\}$ is an eigenvalue of $T^{*}$ with $\operatorname{dim} \operatorname{ker}\left(T^{*}-\lambda I\right) \geq \operatorname{dim} \operatorname{ker} S^{*}$ and therefore $\sigma(T)=\overline{\mathbf{D}}$. Also for every $\lambda$ in $\mathbf{D}$, the operator $T-\lambda I$ is injective. In this note we prove that if the multiplicity of $S\left(=\operatorname{dim} \operatorname{ker} S^{*}\right)$ is finite, then both the approximate point spectrum $\sigma_{\mathrm{ap}}(T)$ and the essential spectrum $\sigma_{\mathbf{e}}(T)$ of $T$ coincide with the unit circle $\partial \mathbf{D}$. This result is applied to show that any analytic Toeplitz operator quasisimilar to a unilateral shift of finite multiplicity must be a unilateral shift. We also characterize contractions which are quasiaffine transforms of unilateral shifts of finite multiplicity in terms of their characteristic functions. This result is similar to the one of $\mathrm{Wu}[\mathbf{1 4}$, Theorem 2.4] which characterizes contractions quasisimilar to unilateral shifts of finite multiplicity.

THEOREM 1. Let $T$ be a contraction such that $T \prec S$, where $S$ is a unilateral shift. If the multiplicity of $S$ is finite, then $\sigma_{\mathrm{ap}}(T)=\sigma_{\mathrm{e}}(T)=\partial \mathbf{D}$ and ind $T=$ ind $S$, where for a Fredholm operator $A$, ind $A$ denotes Fredholm index.

In Theorem 1, if the multiplicity of $S$ is infinite, then the conclusion $\sigma_{\mathrm{ap}}(T)=\partial \mathbf{D}$ need not hold even if $T$ is quasisimilar to $S$ (cf. [2]). Also in this case,

$$
\operatorname{dim} \operatorname{ker}(T-\lambda I)^{*} \geq \operatorname{dim} \operatorname{ker} S^{*}=\infty
$$

for every $\lambda$ in $\mathbf{D}$ and so $\sigma_{\mathbf{e}}(T)=\overline{\mathbf{D}}$.

Received by the editors February 3, 1986 and, in revised form, May 14, 1986.

1980 Mathematics Subject Classification (1985 Revision). Primary 47A45, 47A10.

Key words and phrases. Contraction, quasiaffine transform, unilateral shift, approximate point spectrum, essential spectrum.

(C) 1987 American Mathematical Society $0002-9939 / 87 \$ 1.00+\$ .25$ per page 
Before going to the proof, let us apply Theorem 1 to analytic Toeplitz operators. For $f$ in $H^{\infty}$, the analytic Toeplitz operator $T_{f}$ is the operator of multiplication by $f$ on $H^{2}$, i.e., $T_{f} h=f h$ for $h \in H^{2}$. The analytic Toeplitz operator $T_{f}$ is a unilateral shift if and only if $f$ is a nonconstant inner function, and in this case the multiplicity of $T_{f}$ is $\operatorname{dim}\left(H^{2} \ominus f H^{2}\right)$.

COROLlaRY 1. Let $b$ be a finite Blaschke product and $f \in H^{\infty}$. If $\|f\|_{\infty} \leq 1$ and $T_{f} \prec T_{b}$, then $T_{f}$ is unitarily equivalent to $T_{b}$.

PrOOF. Since $T_{b}$ is a unilateral shift of finite multiplicity and $\left\|T_{f}\right\|=\|f\|_{\infty} \leq 1$, it follows from Theorem 1 that $\sigma_{\mathrm{ap}}\left(T_{f}\right)=\partial \mathrm{D}$ and $\operatorname{dim} \operatorname{ker} T_{f}^{*}=\operatorname{dim} \operatorname{ker} T_{b}^{*}(<\infty)$. Let $M_{f}$ be the operator of multiplication by $f$ on $L^{2}$. Then, since $\sigma\left(M_{f}\right)=\sigma_{\mathrm{ap}}\left(T_{f}\right)$ (see, for example, the proof of [6, Proposition 7.6]), the normal operator $M_{f}$ is unitary and so $f$ is inner. It follows that $T_{f}$ is a unilateral shift and its multiplicity is equal to $\operatorname{dim} \operatorname{ker} T_{b}^{*}$. This shows that $T_{f}$ and $T_{b}$ are unitarily equivalent.

In [4] Conway showed that if $b$ is a single Blaschke factor, i.e., if $T_{b}$ is a unilateral shift of multiplicity one, then any analytic Toeplitz operator quasisimilar to $T_{b}$ must be unitarily equivalent to it. We have the following

COROLLARY 2. Let $b$ be a finite Blaschke product and $f \in H^{\infty}$. If $T_{b} \prec^{i} T_{f} \prec^{i}$ $T_{b}$, then $T_{f}$ and $T_{b}$ are unitarily equivalent.

ProOF. Since $T_{b} \prec^{i} T_{f}$, a result of [5] shows that $f(\mathbf{D}) \subseteq \sigma\left(T_{b}\right)=\overline{\mathbf{D}}$, and so $\|f\|_{\infty} \leq 1$ and $T_{f}$ is a contraction. Then by [10, Proposition 1] the condition $T_{b} \prec^{i} T_{f} \prec^{i} T_{b}$ implies $T_{f} \prec T_{b}$. Therefore the result follows from Corollary 1 .

In the following discussion, we use the functiona: model of Sz.-Nagy and Foias [9] for completely nonunitary contraction. Here a contraction is completely nonunitary (c.n.u.) if there is no nonzero reducing subspace on which it is unitary.

For a (separable) Hilbert space $\mathcal{E}$, let $L^{2}(\mathcal{E})$ and $H^{2}(\mathcal{E})$ denote the spaces of $\mathcal{E}$-valued $L^{2}$ - and $H^{2}$-functions on $\partial \mathrm{D}$, respectively. For two Hilbert spaces $\mathcal{E}$ and $\mathcal{E}^{\prime}$, let $H^{\infty}\left(\mathcal{E}, \mathcal{E}^{\prime}\right)$ denote the space of operator-valued $H^{\infty}$-functions on $\partial \mathrm{D}$ whose values are operators from $\mathcal{E}$ to $\mathcal{E}^{\prime}$. Let $T$ be a c.n.u. contraction, and let $D_{T}=\overline{\operatorname{ran}\left(I-T^{*} T\right)}$ and $D_{T^{*}}=\overline{\operatorname{ran}\left(I-T T^{*}\right)}$. For the characteristic function $\Theta_{T}$ of $T$, which is a contractive operator-valued function in $H^{\infty}\left(D_{T}, D_{T^{*}}\right)$, set

$$
\Delta_{T}\left(e^{i t}\right)=\left(I-\Theta_{T}\left(e^{i t}\right)^{*} \Theta_{T}\left(e^{i t}\right)\right)^{1 / 2} .
$$

Then the (unitarily equivalent) functional model of $T$ is the operator $S\left(\Theta_{T}\right)$ on the Hilbert space

$$
H\left(\Theta_{T}\right)=\left[H^{2}\left(D_{T^{*}}\right) \oplus \overline{\Delta_{T} L^{2}\left(D_{T}\right)}\right] \ominus\left\{\Theta_{T} h \oplus \Delta_{T} h: h \in H^{2}\left(D_{T}\right)\right\}
$$

defined by

$$
S\left(\Theta_{T}\right)(f \oplus g)=P(\chi f \oplus \chi g),
$$

where $\chi\left(e^{i t}\right)=e^{i t}$ and $P$ denotes the orthogonal projection of $H^{2}\left(D_{T^{*}}\right) \oplus \overline{\Delta_{T} L^{2}\left(D_{T}\right)}$ onto $H\left(\Theta_{T}\right)$ (see $[9$, Chapter VI]).

If $T$ is a contraction such that $T \prec S$, where $S$ is a unilateral shift, then it is easily seen that $T$ is of class $C_{\cdot 0}$, that is, $T^{* n} \rightarrow 0$ strongly as $n \rightarrow \infty$, and therefore $T$ is c.n.u. and $\Theta_{T}$ is inner (see [9, Proposition VI.3.5]), i.e., $\Theta_{T}\left(e^{i t}\right)$ 
is isometric a.e. Thus the functional model $S\left(\Theta_{T}\right)$ acts on the space $H\left(\Theta_{T}\right)=$ $H^{2}\left(D_{T^{*}}\right) \ominus \Theta_{T} H^{2}\left(D_{T}\right)$. We also remark the following facts for a c.n.u. contraction $T$ : For every $\alpha \in \mathrm{D}$,

$$
\operatorname{dim} \operatorname{ker}(T-\alpha I)=\operatorname{dim} \operatorname{ker} \Theta_{T}(\alpha), \quad \operatorname{dim} \operatorname{ker}(T-\alpha I)^{*}=\operatorname{dim} \operatorname{ker} \Theta_{T}(\alpha)^{*},
$$

and the left-invertibility of $T-\alpha I$ is equivalent to that of $\Theta_{T}(\alpha)$. In fact, these follow from the facts that for the contraction $T_{\alpha}=(T-\alpha I)(I-\bar{\alpha} T)^{-1}(\alpha \in \mathbf{D})$, there exist unitary operators $A_{\alpha}: D_{T_{\alpha}} \rightarrow D_{T}$ and $B_{\alpha}: D_{T_{\alpha}^{*}} \rightarrow D_{T^{*}}$ such that $T_{\alpha} \mid D_{T_{\alpha}}=$ $-B_{\alpha}^{*} \Theta_{T}(\alpha) A_{\alpha}$ (see $\left[9\right.$, p. 240]), and $T_{\alpha}$ isometrically maps the orthocomplement of $D_{T_{\alpha}}$ onto the one of $D_{T_{\alpha}^{*}}$ (see $[9$, p. 260]).

LEMMA 1. Let $T$ be a c.n.u. contraction with $\operatorname{ker}(T-\alpha I)^{*} \neq\{0\}$ for some $\alpha \in \mathbf{D}$. If $T-\alpha I$ is injective, then $S \prec^{i} T$, where $S$ is a unilateral shift of multiplicity equal to $\operatorname{dim} \operatorname{ker}(T-\alpha I)^{*}$.

ProOF. Let $\mathcal{G}=\operatorname{ker} \Theta_{T}(\alpha)^{*}$ and let $S$ be the unilateral shift on $H^{2}(\mathcal{G})$, i.e., $S f=\chi f, f \in H^{2}(\mathcal{G})$. Since $\operatorname{dim} \mathcal{G}=\operatorname{dim} \operatorname{ker}(T-\alpha I)^{*}$ by a remark given above, it suffices to show $S \prec^{i} S\left(\Theta_{T}\right)$. We define the operator $Y$ from $H^{2}(\mathcal{G})$ to $H\left(\Theta_{T}\right)$ by $Y f=P(f \oplus 0), f \in H^{2}(\mathcal{G})$. It is obvious that $Y S=S\left(\Theta_{T}\right) Y$. To show the injectivity of $Y$, take $f \in \operatorname{ker} Y$. Then $f=\Theta_{T} g$ for some $g \in H^{2}\left(D_{T}\right)$, and so $f(\alpha)=\Theta_{T}(\alpha) g(\alpha) \in \operatorname{ran} \Theta_{T}(\alpha)$. But, since $f \in H^{2}(\mathcal{G})$, we have $f(\alpha) \in \mathcal{G}=$ $\operatorname{ker} \Theta_{T}(\alpha)^{*}$. Hence $f(\alpha)=0$. Since the assumption that $T-\alpha I$ is injective implies the injectivity of $\Theta_{T}(\alpha)$, we also have $g(\alpha)=0$. Therefore $f \in b_{\alpha} H^{2}(\mathcal{G})$ and $g \in$ $b_{\alpha} H^{2}\left(D_{T}\right)$ where $b_{\alpha}\left(e^{i t}\right)=\left(e^{i t}-\alpha\right)\left(1-\bar{\alpha} e^{i t}\right)^{-1}$. Now assume that $f \in b_{\alpha}^{n} H^{2}(\mathcal{G})$ and $g \in b_{\alpha}^{n} H^{2}\left(D_{T}\right)$ for some $n \geq 1$. Then $b_{\alpha}^{-n} f \in H^{2}(\mathcal{G})$ and $b_{\alpha}^{-n} g \in H^{2}\left(D_{T}\right)$. Applying the above argument to the relation $b_{\alpha}^{-n} f=\Theta_{T}\left(b_{\alpha}^{-n} g\right)$, we have $b_{\alpha}^{-n} f \in b_{\alpha} H^{2}(\mathcal{G})$ and $b_{\alpha}^{-n} g \in b_{\alpha} H^{2}\left(D_{T}\right)$. Hence $f \in b_{\alpha}^{n+1} H^{2}(\mathcal{G})$ and $g \in b_{\alpha}^{n+1} H^{2}\left(D_{T}\right)$. Thus we can show by induction on $n$ that $f \in \bigcap_{n \geq 0} b_{\alpha}^{n} H^{2}(\mathcal{G})$, so that $f=0$. This shows that $Y$ is injective and therefore $S \prec^{i} S\left(\Theta_{T}\right)$.

The following lemma is an immediate consequence of the lifting theorem (see [9, Theorem II.2.3 or Theorem VI.3.6]).

LEMMA 2. If $T$ is a contraction and $T \prec S$, where $S$ is the unilateral shift on $H^{2}(\mathcal{E})$, then there exists an outer function $\Phi \in H^{\infty}\left(D_{T^{*}}, \mathcal{E}\right)$ such that $\Theta_{T} H^{2}\left(D_{T}\right)=$ $\left\{f \in H^{2}\left(D_{T^{*}}\right): \Phi f=0\right\}$.

PROOF. The relation $T \prec S$ implies that $T$ is c.n.u. and its functional model $S\left(\Theta_{T}\right)$ acts on $H\left(\Theta_{T}\right)=H^{2}\left(D_{T^{*}}\right) \Theta \Theta_{T} H^{2}\left(D_{T}\right)$. Let $X$ be an injection with dense range such that $X S\left(\Theta_{T}\right)=S X$. By the lifting theorem, there exists $\Phi \in$ $H^{\infty}\left(D_{T^{*}}, \mathcal{E}\right)$ such that $\Phi \Theta_{T}=0$ and $X f=\Phi f$ for $f \in H\left(\Theta_{T}\right)$. Then, since $X$ has dense range, $\Phi$ is outer. The equality $\Theta_{T} H^{2}\left(D_{T}\right)=\left\{f \in H^{2}\left(D_{T^{*}}\right): \Phi f=0\right\}$ follows from $\Phi \Theta_{T}=0$ and the injectivity of $X$.

LEMMA 3. If $T$ is a contraction and $T \prec S$, where $S$ is a unilateral shift of finite multiplicity, then for each $\alpha \in \mathbf{D}$ there exists a scalar function $\delta_{\alpha} \in H^{\infty}$ such that $\delta_{\alpha}(\alpha) \neq 0$ and $\Psi_{\alpha} \Theta_{T}=\delta_{\alpha} I$ for some $\Psi_{\alpha} \in H^{\infty}\left(D_{T^{*}}, D_{T}\right)$.

PrOOF. Let $\mathcal{E}$ be a finite-dimensional Hilbert space with $\operatorname{dim} \mathcal{E}=\operatorname{dim} \operatorname{ker} S^{*}$. Then, since $T$ is a quasiaffine transform of the unilateral shift on $H^{2}(\mathcal{E})$, by Lemma 2 there is an outer function $\Phi \in H^{\infty}\left(D_{T^{*}}, \mathcal{E}\right)$ satisfying the condition in Lemma 2. 
Since $\Phi$ is outer and $\operatorname{dim} \mathcal{E}<\infty$, for $\alpha \in \mathbf{D}$, the operator $\Phi(\alpha)$ is surjective (see [9, Proposition V.2.4]) and so there is a subspace $\mathcal{F} \subseteq D_{T^{*}}$ with $\operatorname{dim} \mathcal{F}=\operatorname{dim} \mathcal{E}$ such that $\Phi(\alpha) \mid \mathcal{F}: \mathcal{F} \rightarrow \mathcal{E}$ is invertible. For $\lambda \in \mathbf{D}$, let $\delta_{\alpha}(\lambda)$ be the determinant of the matrix of $\Phi(\lambda) \mid \mathcal{F}$ with respect to two orthonormal bases in $\mathcal{F}$ and in $\mathcal{E}$. Then $\delta_{\alpha}$ is a function in $H^{\infty}$ with $\delta_{\alpha}(\alpha) \neq 0$ and there is $A_{\alpha} \in H^{\infty}\left(\mathcal{E}, D_{T^{*}}\right)$ such that $\Phi A_{\alpha}=\delta_{\alpha} I$ (see [9, Proposition V.6.1]). Since $\Phi\left(\delta_{\alpha} I-A_{\alpha} \Phi\right)=\left(\delta_{\alpha} I-\Phi A_{\alpha}\right) \Phi=0$, the condition of $\Phi$ implies $\left(\delta_{\alpha} I-A_{\alpha} \Phi\right) H^{2}\left(D_{T^{*}}\right) \subseteq \Theta_{T} H^{2}\left(D_{T}\right)$. Hence, if $\Psi_{\alpha}=\Theta_{T}^{*}\left(\delta_{\alpha} I-A_{\alpha} \Phi\right)$, then, since $\Theta_{T}$ is inner as remarked above, it follows that $\Psi_{\alpha} \in H^{\infty}\left(D_{T^{*}}, D_{T}\right)$ and $\delta_{\alpha} I-A_{\alpha} \Phi=\Theta_{T} \Psi_{\alpha}$. Then, using $\Phi \Theta_{T}=0$, we have $\delta_{\alpha} \Theta_{T}=\left(\delta_{\alpha} I-A_{\alpha} \Phi\right) \Theta_{T}=$ $\Theta_{T} \Psi_{\alpha} \Theta_{T}$. Since $\Theta_{T}$ is inner, $\Psi_{\alpha} \Theta_{T}=\delta_{\alpha} I$.

Proof OF ThEOREM 1. For $\alpha \in \mathbf{D}$, Lemma 3 shows that $\Theta_{T}(\alpha)$ is leftinvertible, hence, as remarked earlier, $T-\alpha I$ is left-invertible. Therefore $\sigma_{\text {ap }}(T) \subseteq$ $\partial \mathbf{D}$. The converse inclusion follows from $\sigma(T)=\overline{\mathbf{D}}$. Thus we have $\sigma_{\mathrm{ap}}(T)=\partial \mathrm{D}$. For $\alpha \in \mathbf{D}$, the relation $T \prec S$ implies $\operatorname{dim} \operatorname{ker}(T-\alpha I)^{*} \geq \operatorname{dim} \operatorname{ker} S^{*}$. But, if $S_{1}$ is a unilateral shift with $\operatorname{dim} \operatorname{ker} S_{1}^{*}=\operatorname{dim} \operatorname{ker}(T-\alpha I)^{*}$, then by Lemma $1 S_{1} \prec^{i} T$, and so $S_{1} \prec^{i} S$. Hence it follows that

$$
\operatorname{dim} \operatorname{ker}(T-\alpha I)^{*}=\operatorname{dim} \operatorname{ker} S_{1}^{*} \leq \operatorname{dim} \operatorname{ker} S^{*}
$$

(see [10, Theorem 5]). Thus we have $\operatorname{dim} \operatorname{ker}(T-\alpha I)^{*}=\operatorname{dim} \operatorname{ker} S^{*}$ for $\alpha \in \mathbf{D}$. This shows that $\sigma_{\mathrm{e}}(T) \subseteq \partial \mathbf{D}$ and ind $(T-\alpha I)=$ ind $S$ for every $\alpha \in \mathbf{D}$. Also, since the point spectrum of a c.n.u. contraction does not intersect with $\partial \mathrm{D}$ (see $\left[\mathbf{9}\right.$, Proposition I.3.1]), we have $\sigma_{\mathbf{e}}(T) \supseteq \sigma_{\mathrm{ap}}(T) \cap \partial \mathbf{D}=\partial \mathbf{D}$. Hence $\sigma_{\mathbf{e}}(T)=\partial \mathbf{D}$. This completes the proof.

In [14], Wu characterized contractions which are quasisimilar to unilateral shifts of finite multiplicity. We have an analogue of this result for contractions which are quasiaffine transfroms of unilateral shifts.

Recall that a contraction $T$ is of class $C_{1}$. if $\lim _{n \rightarrow \infty}\left\|T^{n} x\right\| \neq 0$ for all nonzero $x$, and $T$ is of class $C_{10}$ if it is of class $C_{1}$. and of class $C_{\cdot 0}$.

Proposition 1. Let $T$ be a contraction. Then the following statements are equivalent:

(i) $T \prec S$, where $S$ is a unilateral shift of finite multiplicity.

(ii) $T$ is a contraction of class $C_{10}$ with $\operatorname{dim} \operatorname{ker} T^{*}<\infty$ and there exists a nonzero $\delta \in H^{\infty}$ such that $\Psi \Theta_{T}=\delta I$ for some $\Psi \in H^{\infty}\left(D_{T^{*}}, D_{T}\right)$.

Proof. (i) $\Rightarrow$ (ii) By Theorem 1, $\operatorname{dim} \operatorname{ker} T^{*}=\operatorname{dim} \operatorname{ker} S^{*}<\infty$, and the existence of the function $\delta \in H^{\infty}$ follows from Lemma 3. It is also easily seen that $T$ is of class $C_{10}$.

(ii) $\Rightarrow$ (i) In the case when $\delta$ is outer, this implication is proved in [13, Lemma 1]. Since $T$ is of class $C \cdot 0, T$ is c.n.u. and $H\left(\Theta_{T}\right)=H^{2}\left(D_{T^{*}}\right) \ominus \Theta_{T} H^{2}\left(D_{T}\right)$. We define $X: H\left(\Theta_{T}\right) \rightarrow H^{2}\left(D_{T^{*}}\right)$ by $X f=\left(\delta I-\Theta_{T} \Psi\right) f, f \in H\left(\Theta_{T}\right)$. Since $\left(\delta I-\Theta_{T} \Psi\right) \Theta_{T}=0$, it follows that $X S\left(\Theta_{T}\right)=S X$, where $S$ is the unilateral shift on $H^{2}\left(D_{T^{*}}\right)$. If $X f=0$, then $\delta f=\Theta_{T} \Psi f$ and so $\delta\left(S\left(\Theta_{T}\right)\right) f=P(\delta f)=0$. Since $\delta \neq 0$, this shows $S\left(\Theta_{T}\right)^{n} f \rightarrow 0$ strongly $(n \rightarrow \infty)$ (see [9, Proposition III.4.1]), and since $T$ is of class $C_{1} ., f=0$. Thus $X$ is injective, and therefore $T$ is a quasiaffine transform of the unilateral shift $S \mid \overline{\operatorname{ran} X}$, whose multiplicity is equal to $\operatorname{dim} \operatorname{ker} T^{*}<\infty$ by Theorem 1 . This proves (i).

For two operators $T_{1}$ on $\mathscr{H}_{1}$ and $T_{2}$ on $\mathscr{H}_{2}, T_{1} \prec^{c i} T_{2}$ denotes that there is a family $\left\{X_{\alpha}\right\}$ of injections $X_{\alpha}: \mathscr{H}_{1} \rightarrow \mathscr{H}_{2}$ such that $X_{\alpha} T_{1}=T_{2} X_{\alpha}$ and $\bigvee_{\alpha} \operatorname{ran} X_{\alpha}=\not_{2}$ 
We write $T_{1} \sim^{c i} T_{2}$ if $T_{1} \prec^{c i} T_{2}$ and $T_{2} \prec^{c i} T_{1}$. In [10], this relation $T_{1} \sim^{c i} T_{2}$ is called completely injection-similarity. [10, Proposition 3] shows that the relations $T \prec S$, where $T$ is a contraction and $S$ is a unilateral shift, need not imply the quasisimilarity of $T$ and $S$.

PROPOSITION 2. Let $T$ be a contraction and $S$ be a unilateral shift (whose multiplicity may be infinite). Then the relation $T \prec S$ is equivalent to $T \sim^{c i} S$.

ProOF. That $T \sim^{c i} S$ implies $T \prec S$ was proved in [10, Proposition 1]. To show the converse implication, we have to prove $S \prec^{c i} T$. Let $\left\{\alpha_{n}\right\}$ be a sequence of distinct complex numbers such that $\alpha_{n} \in \mathbf{D}$ and $\lim _{n \rightarrow \infty} \alpha_{n}=0$. For each $n$, define $Y_{n}: H^{2}\left(\operatorname{ker} \Theta_{T}\left(\alpha_{n}\right)^{*}\right) \rightarrow H\left(\Theta_{T}\right)$ by $Y_{n} f=P f$. Since the asumption $T \prec S$ implies the injectivity of $T-\alpha_{n} I$, as shown in the proof of Lemma $1, Y_{n}$ is an injection and $Y_{n} S_{n}=S\left(\Theta_{T}\right) Y_{n}$, where $S_{n}$ is the unilateral shift on $H^{2}\left(\operatorname{ker} \Theta_{T}\left(\alpha_{n}\right)^{*}\right)$. If $\operatorname{dim} \operatorname{ker} S^{*}<\infty$, then by Theorem 1 we have $\operatorname{dim} \operatorname{ker} \Theta_{T}\left(\alpha_{n}\right)^{*}=\operatorname{dim} \operatorname{ker} S^{*}$, and the equality also holds in case $\operatorname{dim} \operatorname{ker} S^{*}=\infty$, because the relation $T \prec S$ implies dim $\operatorname{ker} \Theta_{T}\left(\alpha_{n}\right)^{*}=\operatorname{dim} \operatorname{ker}\left(T-\alpha_{n} I\right)^{*} \geq \operatorname{dim} \operatorname{ker} S^{*}$. Thus $S_{n}$ is unitarily equivalent to $S$, and therefore to prove $S \prec^{c i} T$, we have only to show $\bigvee_{n} \operatorname{ran} Y_{n}=$ $H\left(\Theta_{T}\right)$. Suppose that $f$ in $H\left(\Theta_{T}\right)$ is orthogonal to all $\operatorname{ran} Y_{n}$. The orthogonality of $f$ to $\operatorname{ran} Y_{n}$ implies that $f\left(\alpha_{n}\right)$ is orthogonal to $\operatorname{ker} \Theta_{T}\left(\alpha_{n}\right)^{*}$, that is, $f\left(\alpha_{n}\right) \in$ $\overline{\operatorname{ran} \Theta_{T}\left(\alpha_{n}\right)}$. We now use the function $\Phi$ given in Lemma 2. Since $\Phi \Theta_{T}=0$, we have $\Phi\left(\alpha_{n}\right) \Theta_{T}\left(\alpha_{n}\right)=0$ and therefore $\Phi\left(\alpha_{n}\right) f\left(\alpha_{n}\right)=0$ for all $n$. It follows from the uniqueness theorem for an operator-valued analytic function that $\Phi f=0$, and so $f \in \Theta_{T} H^{2}\left(D_{T}\right)$. Then, since $f \in H\left(\Theta_{T}\right)$, we have $f=0$. This shows $\bigvee_{n} \operatorname{ran} Y_{n}=H\left(\Theta_{T}\right)$.

\section{REFERENCES}

1. H. Bercovici and K. Takahashi, On the reflexivity of contractions on Hilbert space, J. London Math. Soc. (2) 32 (1985), 149-156.

2. W. S. Clary, Equality of spectra of quasisimilar hyponormal operators, Proc. Amer. Math. Soc. 53 (1975), 88-90.

3. J. B. Conway, Subnormal operators, Research Notes in Math., vol. 51, Pitman, Boston, Mass., 1981.

4. __ On quasisimilarity for subnormal operators. II, Canad. Math. Bull. 25 (1982), 37-40.

5. J. A. Deddens, Intertwining analytic Toeplitz operators, Michigan Math. J. 18 (1971), 243-246.

6. R. G. Douglas, Banach algebra techniques in operator theory, Pure and Appl. Math., vol. 49, Academic Press, New York, 1972.

7. W. W. Hastings, Subnormal operators quasisimilar to an isometry, Trans. Amer. Math. Soc. 256 (1979), 145-161.

8. B. Sz.-Nagy, Diagonalization of matrices over $H^{\infty}$, Acta Sci. Math. 38 (1976), 223-238.

9. B. Sz.-Nagy and C. Foias, Harmonic analysis of operators on Hilbert space, North-Holland, Amsterdam, 1970.

10. _ Jordan model for contractions of class $C_{\cdot 0}$, Acta Sci. Math. 36 (1974), 305-322.

11. K. Takahashi, Contractions with the bicommutant property, Proc. Amer. Math. Soc. 93 (1985), 91-95.

12. M. Uchiyama, Contractions and unilateral shifts, Acta Sci. Math. 46 (1983), 345-356.

13. P. Y. Wu, When is a contraction quasisimilar to an isometry?, Acta Sci. Math. 44 (1982), 151-155.

14. __ Contractions quasisimilar to an isometry, preprint.

Department of Mathematics, Sapporo Medical College, Minami-1 Nishi-17, SAPPORO, JAPAN 